\title{
WAB*: A Quantitative Metric Based on WAB
}

\author{
Ana Belén Martínez, Aquilino A. Juan, Darío Álvarez, and Mª del Carmen Suárez \\ University of Oviedo, Department of Computing, \\ Calvo Sotelo s/n 33007 Oviedo, Spain \\ \{belenmp, aajuan, darioa, macamen\} @uniovi.es
}

\begin{abstract}
Web accessibility metrics are crucial for the quantitative evaluation of web sites. We present a new automatic metric called WAB, based on the WAB metric with extensions inspired from the UWEM metric. The first results are encouraging as we have obtained better precision when calculation the accessibility level measured for a site.
\end{abstract}

Keywords: Accessibility, Metrics, Evaluation.

\section{Introduction}

We need quantitative evaluations of accessibility [1] to compare accessibility levels of two sites (or for different versions of the same site). Quantitative evaluations are also needed to analyze the evolution over time of accessibility with the changes done during the life of the site (monitorization). For these measurements we can use automatic metrics (no human judgment is involved to calculate an accessibility score) such as WAB [2] or WAQM [1], or semi-automatic, such as Failure_Rate [3] or UWEM [4].

We focused on two of these metrics: WAB and UWEM. The first one is popular in accessibility studies [5] because it is fully automatic. We focused on the second because it is being sponsored by the European Union as part of the Unified Web Evaluation Methodology to evaluate accessibility (EIAO Project [6][7]). We performed some studies using these metrics [8][9] using our own flexible tool called Iris that performs the quantitative evaluation of web sites using different metrics. These studies were able to discover deficiencies in each metric that we tried to solve. Such is the goal of this work: to propose a new metric called WAB*, fully automated, which is based on the WAB metric with some UWEN-like extensions, that retains the automatic feature of $\mathrm{WAB}$ and improves the precision of the accessibility score using more detailed checkpoints.

\section{Related Work}

The following is a brief summary of the features of the two metrics considered: WAB and UWEM. 


\subsection{Web Accessibility Barrier (WAB)}

WAB metric [10] yields a quantitative score that looks at 25 checkpoints based on WCAG 1.0 (5 of Priority 1, 13 of Priority 2 and 7 of Priority 3). The number of violations of the checkpoints is the basis for the score:

$$
\text { WAB score }=\frac{\sum_{p} \sum_{v}\left(\frac{n_{v}}{N_{v}} \times W_{v}\right)}{N_{p}}
$$

With $p=$ Total pages of a Website, $v=$ Total violations of a Web page, $n v=$ Number of violations, $N v=$ Number of potential violations, $W v=$ Weight of violations in inverse proportion to WCAG priority level, and $N p=$ Total number of pages checked.

WAB scores greater than 5.5 denote a web site with serious accessibility problems [2]. A score of zero denotes that the Web site does not violate any Web accessibility guideline and should not present any accessibility barrier to persons with disabilities.

\subsection{Unified Web Evalution Methodology (UWEM)}

The UWEM [11] describes a methodology for evaluating conformance of Web sites with the WCAG 1.0 guidelines. Currently it is designed to be conformant with WCAG 1.0 priority 1 and 2 checkpoints, and provides manual and automatic evaluation. For automatic evaluation, it applies a set of checkpoints marked as "fully automatable" by the methodology.

One of the outputs is a score computed using the following metric:

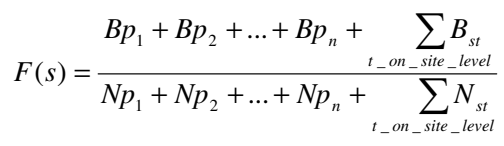

With $F(s)=$ UWEM score for the site s, Bpi = Total number of "fail" results from all tests within page pi and $\mathrm{Npi}=$ Total number applications of all tests within page $p i$. Bearing in mind that some UWEM tests apply to the web site as a whole and not to individual pages, $B s t=$ Total number of "fail" results from all tests on site level, Nst= Total number applications of all tests on site level.

The UWEM metric $(F s)$ value is a number between 0 and 1. A score of zero denotes that the Web site should not present serious problems of accessibility.

\section{$3 \mathbf{W A B}^{*}$}

As mentioned before, the WAB metric evaluates 25 checkpoints from the 3 priority levels. However, the tests performed to evaluate the checkpoints are sometimes vague when specifying the way to determine the number of potential violations of each checkpoint. This is owed mostly to the evolution of the technology used in the construction of web sites. Table 1 shows some examples. 
Table 1. Some of the most vague checkpoints in the WAB metric

\begin{tabular}{ll}
\hline CheckPoint & Number of potential violations \\
\hline Each frame should reference a HTML file & Every $<$ frame $>\rightarrow$ does not include iframe \\
$\begin{array}{l}\text { Each frame should have a title } \\
\begin{array}{l}\text { Do not use the same link sentence when pointing } \\
\text { to different URLs }\end{array}\end{array}$ & $\begin{array}{l}\text { Every }<\text { frame }>\rightarrow \text { does not include }<\text { iframe }> \\
\text { Use relative position and size instead of absolute }>\rightarrow \text { does not include }<\text { area }>\end{array}$ \\
\hline $\begin{array}{l}\text { Every }<\text { table }>,<\text { th }>,<\text { dd }>,<\text { frame }>\rightarrow \text { does not } \\
\text { include }<\text { div }>\text { or }<\text { iframe }>\end{array}$ \\
\hline
\end{tabular}

On the other hand, the UWEM metric specifies priority 1 and 2 checkpoints of greater precision than the ones used in WAB (as seen in Table 2) when identifying the number of potential violations. However, the downside of this metric is that no priority 3 checkpoint is used.

Table 2. Some important checkpoints of the WAB metric

\begin{tabular}{|c|c|}
\hline CheckPoint & Number of potential violations \\
\hline Each frame should reference an HTML file & Every $<$ frame $>$, <iframe $>$ \\
\hline Each frame should have a title & Every $<$ frame $>$, <iframe $>$ \\
\hline $\begin{array}{l}\text { Do not use the same link sentence when pointing } \\
\text { to different URLs }\end{array}$ & Every $<a>,<$ area $>$ \\
\hline Use relative position and size instead of absolute & $\begin{array}{l}\text { Every }<\text { table }>,<\text { th }>,<\text { td }>,<\text { frame }>\text {, <iframe }>\text {, } \\
<\text { div }>,<\text { object }>\text {, < applet }>,<\text { frameset }>\end{array}$ \\
\hline Val & Every CSS \\
\hline Do not use CSS rules that produce blinking & Every CSS \\
\hline
\end{tabular}

Taking into account these deficiencies, we have developed $\mathrm{WAB}^{*}$ extension of the WAB metric. A summary of the most important features follows:

1. WAB* considers all 3 priority levels (as WAB), and not only 2 .

2. $\mathrm{WAB}^{*}$ uses the same WAB metric (with the same weights).

3. $\mathrm{WAB}^{*}$ has all $\mathrm{WAB}$ checkpoints but updated to eliminate deficiencies. For example:

a. Not only frames, but iframes are tested.

b. Not only table, th, td, and frame are tested for relative size and position, but also div, iframe, object, and applet.

4. Besides, $\mathrm{WAB}^{*}$ includes eleven automatic checkpoints from the UWEM metric.

The final result is a metric with 36 fully automatic, evaluable checkpoints. 6 are priority 1,23 are priority 2 , and 7 are priority 3 . The complete table is not included for space reasons.

\section{A Practical Case: Evaluating the Accessibility of the European Banking Sector}

We performed an accessibility study of the European Banking Section (some results are published in [9]) using the Iris tool previously mentioned. Among other goals, we 
evaluated the behaviour of the new $\mathrm{WAB} *$ metric with relation to WAB and UWEM. We took the Dow Jones EUROSTOXX TMI Banks list and selected 51 banks belonging to 11 countries. The tool allowed us to evaluate 30,600 pages ( 51 sites $\mathrm{X}$ 200 pages in each site in 3 different depth levels $x 3$ metrics). The conclusions drawn from the study state that the new $\mathrm{WAB}^{*}$ metric results are alongside $\mathrm{WAB}$ and UWEM, but the scores are more detailed, and always higher than WAB, mostly because of more exhaustive tests included.

\section{Conclusions}

We have introduced $\mathrm{WAB}^{*}$, a new fully-automatic metric. It is based on WAB but has a much more severe accessibility evaluation, using also UWEM-inspired checkpoints. It evaluates 36 checkpoints against WAB's 25 , and with more precision. The first results from studies performed using the new metric show it provides more precise markers for accessibility levels than the other two metrics.

\section{References}

1. Vigo, M., Arrue, M., Brajnik, G., Lomuscio, R., Abascal, J.: Quantitative Metrics for Measuring Web Accessibility (W4A), pp. 99-107. ACM Press, New York (2007)

2. Hackett, S., Parmanto, B., Zeng, X.: Accessibility of Internet Websites through Time. In: ACM SIGACCESS Accessibility and Computing, pp. 32-39 (2003)

3. Sullivan, T., Matson, R.: Barriers to Use: Usability and Content Accessibility on the Web's Most Popular Sites. In: Conference on Universal Usability, pp. 139-144. ACM Press, New York (2000)

4. Wab Cluster. Unified Web Evaluation Methodology (UWEM), http: / / www.wabcluster.org/deliverables.html

5. Freire, A.P., Bittar, T.J., Fortes, R.P.: An Approach Based on Metrics for Monitoring Web Accessibility in Brazilian Municipalities Web Sites. In: ACM Symposium on Applied Computing, pp. 2421-2425 (2008)

6. European Internet Accessibility Observatory (EIAO), http : / www. eiao.net/

7. Holmesland, M.S., Ulltveit-Moe, N., Balachandran, A., Goodwin, M.: A Proposed Architecture for Large Scale Web Accessibility Assessment. In: Miesenberger, K., Klaus, J., Zagler, W.L., Karshmer, A.I. (eds.) ICCHP 2006. LNCS, vol. 4061, pp. 234-241. Springer, Heidelberg (2006)

8. De Andres, J., Lorca, P., Martínez, A.B.: Social Responsability versus Efficiency Gains: Which are the Factors that Underlie in the Implementation of Web Accessibility by Listed Firms? In: IASK E-Activity and Leading Technologies 2008 \& InterTIC, pp. 49-58 (2008)

9. De Andrés, J., Lorca, P., Martínez, A.B.: Economic and Financial Factors for the Adoption and Visibility Effects of Web Accessibility. The Case of European Banks. Journal of the American Society for Information Science and Technology (accepted, 2009) (publication pending)

10. Parmanto, B., Zeng, X.: Metric for Web Accessibility Evaluation. Journal of the American Society for Information Science and Technology 56(13), 1394-1404 (2005)

11. Nietzio, A., Strobbe, C., Velleman, E.: TheUnified Web Evaluation Methodology (UWEM) 1.2 for WCAG 1.0. In: Miesenberger, K., Klaus, J., Zagler, W.L., Karshmer, A.I. (eds.) ICCHP 2008. LNCS, vol. 5105, pp. 394-401. Springer, Heidelberg (2008) 